\title{
Correlation of Exercise Capacity with Functional Disability in Patients with Osteoarthritis of Knee
}

Swati V. Kubal ${ }^{1}$, Kshitija S. Ghole ${ }^{* 2}$.

${ }^{1}$ Assistant Professor, Department of Physiotherapy, P.T. School and Centre, T.N.M.C, Mumbai, Maharashtra, India.

${ }^{* 2}$ Department of Physiotherapy, P.T. School and Centre, T.N.M.C, Mumbai, Maharashtra, India.

\section{ABSTRACT}

Introduction: Osteoarthritis of knee is a progressive joint disease which produces pain, inflammation and destruction of joint which in turn leads to range of motion limitation and walking disabilities. Affection in physical functioning is determined not only by just activities involving lower limb capacity but also by the activities which require use of upper extremities. Hence in this study, 6MWD and no. of rings moved in 6 minutes were taken as an outcome measures for determining the exercise capacity. KOOS is a self-administered questionnaire which was used in its cross culturally adapted format for determining the functional disability in patients.

Objectives: 1 . To study correlation of exercise capacity with functional disability in patients with osteoarthritis of knee. 2. To study correlation of upper limb exercise capacity with lower limb exercise capacity in patients with osteoarthritis of knee.

Methods: Cross sectional, observational study including 30 patients of either gender having unilateral osteoarthritis of knee conducted in a tertiary care hospital.

Results: No. of rings moved in 6 minutes and global KOOS score showed no statistically significant correlation. 6MWD and global KOOS score showed no statistically significant correlation. 6 minute walk distance and 6 minute peg board ring test showed no statistically significant correlation.

Conclusion: The study suggests that there is no correlation of exercise capacity with functional disability in patients with osteoarthritis of knee. Also, there is no correlation found between upper limb exercise capacity and lower limb exercise capacity in patients with osteoarthritis of knee.

KEY WORDS: Knee osteoarthritis, Knee pain, squat depth, functional disability, cardiorespiratory endurance, Knee Injury and Osteoarthritis Outcome Score, Quality of life.

Address for correspondence: Kshitija Santosh Ghole, Department of Physiotherapy, P.T. School and Centre, T.N.M.C, Mumbai, Maharashtra, India. Mobile no. 8767906586 / 8369040983 E-Mail: kshitighole@gmail.com

\section{Quick Response code}

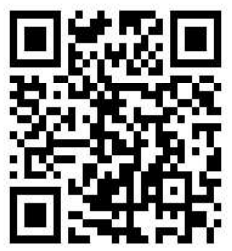

DOI: $10.16965 /$ ijpr.2021.136 Journal Information

(2)

Internation
ISSN (E) 2321-1822 | ISSN (P) 2321-8975 https://www.ijmhr.org/ijpr.html

DOI-Prefix: https://dx.doi.org/10.16965/ijpr

\section{(cc) BT-Niv-Bi}

\section{Article Information}

$\begin{array}{ll}\text { Received: } 06 \text { May } 2021 & \text { Accepted: 14 Jun } 2021 \\ \text { Peer Review: 09 May 2021 } & \text { Published (O): } 11 \text { Jul } 2021 \\ \text { Revised: None } & \text { Published (P): } 11 \text { Aug } 2021\end{array}$

\section{INTRODUCTION}

Osteoarthritis is a chronic degenerative disorder primarily affecting the articular cartilage of synovial joint with eventual bony remodeling and overgrowth at margins of the joint. There is also progression of synovial and capsular thickening and joint effusion. It mainly affects the elderly as elderly lose the water retention ability more acutely and produce proteoglycans which leads to degenerative changes such as osteoarthritis. Osteoarthritis is a progressive joint disease which produces pain, inflammation and destruction of joint which in turn leads to 
Swati V. Kubal, Kshitija S. Ghole. Correlation of exercise capacity with functional disability in patients with osteoarthritis of knee.

range of motion limitation and walking disabilities. Symptoms of osteoarthritis of knee such as pain and stiffness of joint, impaired muscle strength of the limb, lead to restricted locomotion activity and reduced quality of life [1].

6 min walk test is a self-paced exercise testing which assesses submaximal level of physical functioning. Distance walked in 6 minutes reflects functional capacity required for ADLs. It is easy to perform and is better tolerated. According to ATS guidelines, the distance walked by patients on flat, hard surface quickly in 6 minutes is measured and documented as 6 minute walked distance (6MWD). Global and integrated response of all systems (cardiovascular and pulmonary system, systemic and peripheral circulation, blood, neuromuscular units and muscle metabolism) instead of specific information on function of each different organ and systems. Submaximal levels of exertion are required for performing most activities of daily living thus 6MWD may better reflect functional exercise level for daily physical activities. It is also used as a predictor of morbidity and mortality along with being a one-time measure of functional status of patients $[2,3]$.

Physical function is affected not only during performing walking and stair climbing but also while performing simple activities of daily living which involve upper extremities. Either supported or unsupported arm activities such as combing hair, doing dishes, brushing teeth shaving, putting things on overhead shelves are important components of activities of daily living. Literature has demonstrated that metabolic demands are increased in healthy adults with simple arm movements. 6 minute peg board ring test mimics activities of daily living which require unsupported arm function. It is inexpensive and simple test which assesses both arm function and endurance [4-6].

Knee injury and osteoarthritis outcome score (KOOS) is a self-administered questionnaire which has 42 items and 5 separately scored subscales which assesses pain, symptoms like swelling and range of motion limitation, activities of daily living, sport and recreation function and knee related quality of life [7]. It evaluates both short term and long term effects of knee injury. Scores are transformed from 0 to 100 scale, 0 represents extreme knee problems and 100 represents no knee problems. It also has been cross culturally adapted in Hindi and Marathi along with its validation.

Future limitations in patients with osteoarthritis of hip and knee have avoidance of physical activities as a risk factor. In order to prevent feared or actual pain, patients tend to avoid physical activities which results in muscle weakness. As osteoarthritis is frequently activity related this strategy acts as adaptive coping strategy. If this is continued till long term then it is hypothesized that it might lead to deterioration of muscle strength which will in turn result in greater activity limitation [8].

There are studies available which shows that cardiorespiratory endurance is affected in patients with osteoarthritis of knee along with reduction in their quality of life [9]. But there are few studies which show relationship between upper limb exercise capacity and lower limb exercise capacity in osteoarthritis of knee patients. 6MWT incorporates walking for 6 minutes which is one of the painful activities of daily living for patients with osteoarthritis of knee. Thus the purpose of this study was to find out the correlation of exercise capacity with functional disability in patients with osteoarthritis of knee and to study correlation of upper limb exercise capacity with lower limb exercise capacity in patients with osteoarthritis of knee.

\section{METHODOLOGY}

The study was conducted in physiotherapy department of TNM College and BYL Nair Charitable hospital, Mumbai with:

\section{Inclusion criteria:}

1. Subjects of either gender between the ages 50 to 60 yrs.

2. Subjects having less than or equal to grade 2 osteoarthritis according to Kellgren and Lawrence grading system.

3. Subjects with unilateral osteoarthritis.

4. Pain on VAS less than $7 / 10$. 
Swati V. Kubal, Kshitija S. Ghole. Correlation of exercise capacity with functional disability in patients with osteoarthritis of knee.

5. Subjects who were able to understand the procedure.

6. Subjects who were willing to participate in the study.

\section{Exclusion criteria:}

1. Subjects with cardiorespiratory involvement.

2. Subjects with neurological involvement.

3. Subjects with any other musculoskeletal involvement.

\section{Subjects using walking aid.}

The Institutional Ethical Committee of TNM College and BYL Nair Charitable hospital, Mumbai had approved the research protocol.

Design: Present study is Cross - sectional, Observational study conducted in the physiotherapy department of a tertiary care hospital with 30 Patients with knee osteoarthritis, patients were recruited using the convenient, periodic sampling technique.

Method: Patients coming in physiotherapy department of TNM College and BYL Nair Charitable hospital, Mumbai with diagnosed osteoarthritis were selected according to the inclusion and exclusion criteria. Written consent was taken from the patients. The purpose and procedure of the study was informed to the patients in the language best understood by them. Anthropometric data was collected.

Patients were evaluated according to the case record form. Patients were asked to perform 6 minute peg board and ring test. After 6 minute peg board and ring test patients were administered with KOOS questionnaire in the language best understood by them. Patients' choices of answer were marked accordingly which required around 10 to 15 minutes and also served as a rest period for the patients. After 6 minute peg board and ring test patients were given 10 to 15 minutes of a rest gap and were asked to perform 6 minute walk test.

KOOS: Knee injury and osteoarthritis outcome score (KOOS) is a self-administered questionnaire which has 42 items and 5 separately scored subscales which assesses pain, symptoms like swelling and range of motion limitation, activities of daily living, sport and recreation function and knee related quality of life. [7] It evaluates both short term and long term effects of knee injury. Scores are transformed from 0 to 100 scale, 0 represents extreme knee problems and 100 represents no knee problems. It also has been cross culturally adapted in Hindi and Marathi along with its validation.

6 minutes peg board and ring test: Subjects were made to sit comfortably in the chair. Peg board was mounted on the wall. Rings were of $50 \mathrm{gms}$ weight each and there were 2 higher and 2 lower pegs. Subjects were asked to move as many rings as possible from one point to the other point in given 6 minutes. Scoring was done by the number of rings moved during 6 minute period. Oxygen saturation was measured using SpO2 probe attached to the subject's great toe [5-7].

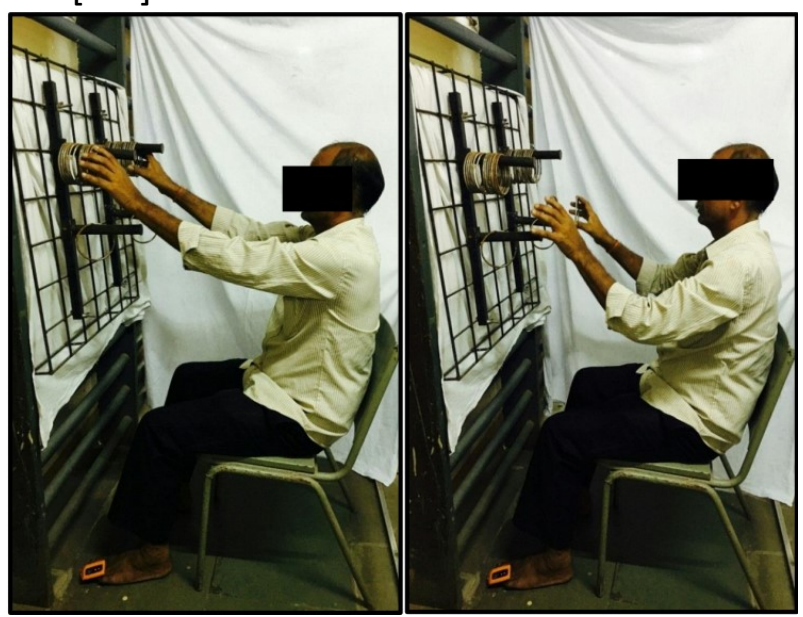

Fig. 1: 6 minute peg board and ring test

6 min walk test: It was conducted according to the ATS guidelines. Target HR was calculated for the subject by using the formula (Target $\mathrm{HR}=$ resting $\mathrm{HR}+(0.6$ (HRmax - resting HR)) $($ HRMax $=206.9-$ age $\times 0.67) .30$ meter walkway was measured by using a measuring tape and the distance was demarcated by cones. Before starting the test, subjects were made to sit on the chair for 5 minutes comfortably and their heart rate was recorded by attaching a pulse oximeter to their index finger. This was recorded as HR at rest.

Subjects were then asked to walk on a 30 meter walkway for 6 minutes at a comfortable speed. The distance covered by subjects was measured in laps. Termination criteria of the test was if 1 . Target Heart Rate was achieved 2.If subject complained of fatigue and 3 .If subject wanted to terminate the test secondary to 
Swati V. Kubal, Kshitija S. Ghole. Correlation of exercise capacity with functional disability in patients with osteoarthritis of knee.

pain. Rate of perceived exertion was checked using Modified Borg scale. [ ${ }^{3]}$ ( $R=0.88$ to 0.94, validity $=0.71$ to 0.82 good reliability and moderate validity)

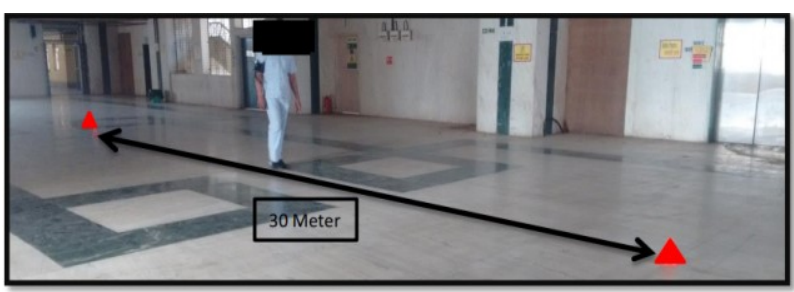

Fig. 2: 6 minute walk test.

Statistical analysis: All statistical analyses were performed using the SPSS version 16.0 (IBM, Armonk, NY, USA) for Windows. Alpha level was set at 0.05 to control for Type I error, and confidence interval was set at $95 \%$ for all statistical analysis. As data did not pass normality, Spearman $r$ correlation test was used.

\section{RESULTS}

The total no. of participants in the study was 30 out of which 20 were females and 10 were males. The mean age of the participants in the group was $53.60 \pm 2.93$ with a mean BMI of $27.79 \pm 3.92$.

In the study patients had mean \pm SD of distance walked in 6 minutes as $364.50 \pm 32.75$ meters and $281.87 \pm 28.00$ as the number of rings moved in 6 minutes. Median for distance walked in 6 minutes by patients in this study was 360 meters and number of rings moved in 6 minutes was 280. Using a spearman's test on the data we found no correlation $(r=0.109$, $\mathrm{N}=30$ ) between 6 minute walk distance and global KOOS score. Using a spearman's test on the data we found no correlation $(r=-0.102, N$ $=30$ ) between no. of rings moved and global KOOS score. Using a spearman's test on the data we found no correlation $(r=0.101$, $\mathrm{N}=30$ ) between 6 minute walk distance and no. of rings moved in 6 minutes.

\section{DISCUSSION}

KOOS is a self-reported questionnaire which talks mainly about functional status of patients with osteoarthritis of knee along with quality of life. Mean global KOOS score of patients in our study was $49.91 \%$ which denotes around moderate affection in patients' lives due to osteoarthritis of knee.
Many factors such as emotional status of patients, social participation by patients, psychological well-being of patients, etc. play an important role while the patients are answering questions which may explain the subjectivity of answers to the questions asked ${ }^{[10]}$ Thus to avoid this factor 6MWT and 6PBRT were assessed to evaluate energy expenditure and exercise capacity of patients with osteoarthritis of knee.

Factors such as weight bearing pain, joint instability sometimes mask patient's functional capacity since 6MWT incorporates weight bearing activity and increased weight bearing in turn increases pain [11]. Therefore 6 PBRT which uses involvement of upper limbs, was used to assess exercise capacity in patients with OA knee so that weight bearing pain which is one of the symptoms of OA knee was not the limiting factor in testing cardiovascular endurance [11].

\section{CONCLUSION}

In current study we found no correlation which may be because the age group involved in the study belonged to physically active middle-aged adults and also pain experienced by patients which is the main factor in limiting lower limb exercise capacity was moderate. This means that for current study population general exercise capacity was independent of the knee pain experienced by patients.

In the current study we could not comment on which exercise capacity was more affected since we did not have normative values of 6PBRT. Thus, we found no correlation between exercise capacity with fuctional disability in patients with osteoarthritis of knee. Also, we found no correlation between upper limb and lower limb exercise capacity in patients with osteoarthritis of knee.

Further research can be carried out with same objectives in different age groups and with people having a grade of $>2$ on $K \& L$ grading system.

Further detailed studies can be carried out to confirm our findings regarding the effect of osteoarthritis of knee on overall exercise capacity and cardiovascular endurance of 
Swati V. Kubal, Kshitija S. Ghole. Correlation of exercise capacity with functional disability in patients with osteoarthritis of knee.

patients. Also upper limb exercise energy expenditure can be assessed in OA knee patients.

\section{Conflicts of interest: None}

\section{REFERENCES}

[1]. Ivan Luis Andrade, Martha Cavalcante Castro, Carla Daltro, and Marcos Almeida Matos Quality of life and functional independence in patients with osteoarthritis of knee. Knee Surgery And Related Research 2016;28(3):219-24.

[2]. American Thoracic Society, ATS statement: Guidelines for the six minute walk test. J Respir Crit Care Med. 2002;166:111-7.

[3]. Roberta E. Rikli and C. Jessie Jones, The reliability and validity of 6 min walk test as a measure of physical endurance in older adults. Journal of $\mathrm{Ag}$ ing and Physical Activity, 1998;(6(4):363-375.

[4]. Karina Maria Cancelliero-Gaiad, Daniela Ike, Liliana Soave,_Evelim Leal de Freitas Dantas Gomes, Fernanda Dultra Dias, Dirceu Costa, Correaltion between functional capacity and health related quality of life in COPD, A case series. Fisioter. Mov., Curitiba, 2014;27(4):505-514.

[5]. Janaudis-Ferreira T, Beauchamp MK, Goldstein RS, Brooks D. How should we measure arm exercise capacity in patients with COPD? A systematic review. Chest. 2012 Jan;141(1):111-120. doi: 10.1378/chest.11-0475. Epub 2011 Jun 9. PMID: 21659430.

[6]. Lima VP, Almeida FD, Janaudis-Ferreira T, Carmona B, Ribeiro-Samora GA, Velloso M. Reference values for the six-minute pegboard and ring test in healthy adults in Brazil. J Bras Pneumol. 2018 MayJun;44(3):190-194. doi: 10.1590/S180637562017000000388. PMID: 30043884; PMCID: PMC6188694.
[7]. Collins NJ, Prinsen CA, Christensen R, Bartels EM, Terwee CB, Roos EM. Knee Injury and Osteoarthritis Outcome Score (KOOS): systematic review and meta-analysis of measurement properties. Osteoarthritis Cartilage. 2016 Aug;24(8):1317-29. doi: 10.1016/j.joca.2016.03.010. Epub 2016 Mar 21. PMID: 27012756.

[8]. Steultjens MP, Dekker J, Bijlsma JW. Avoidance of activity and disability in patients with osteoarthritis of the knee: the mediating role of muscle strength. Arthritis Rheum. 2002 Jul;46(7):1784-8. doi: 10.1002/art.10383. PMID: 12124862.

[9]. Edward F. Philbin, Gerald D. Michael and Thomas E. Miller Cardiovascular fitness and health in patients with end stage osteoarthritis. Arthritis and Rheumatism, 1995;38(6):799-805.

[10]. Akhavan NS, Ormsbee L, Johnson SA, George KS, Foley EM, Elam ML, Ezzat-Zadeh Z, Panton LB, Arjmandi $\mathrm{BH}$. Functionality in Middle-Aged and Older Overweight and Obese Individuals with Knee Osteoarthritis. Healthcare (Basel). 2018 Jul 4;6(3):74. doi: 10.3390/healthcare6030074. PMID: 29973574; PMCID: PMC6165234.

[11]. Gomes-Neto $M$, Araujo AD, Junqueira ID, Oliveira D, Brasileiro A, Arcanjo FL. Comparative study of functional capacity and quality of life among obese and non-obese elderly people with knee osteoarthritis. Rev Bras Reumatol Engl Ed. 2016 MarApr;56(2):126-30. English, Portuguese. doi: 10.1016/j.rbre.2015.08.014. Epub 2015 Sep 26. PMID: 27267525.

\section{How to cite this article:}

Swati V. Kubal, Kshitija S. Ghole. Correlation of exercise capacity with functional disability in patients with osteoarthritis of knee. Int J Physiother Res 2021;9(4):3883-3887. DOI: 10.16965/ijpr.2021.136 